\title{
TOPIC MODELLING FOR OBJECT-BASED CLASSIFICATION OF VHR SATELLITE IMAGES BASED ON MULTISCALE SEGMENTATIONS
}

\author{
Li SHEN $^{\mathrm{a}, \mathrm{b} *}$, Linmei WU ${ }^{\mathrm{b}}$, Zhipeng LI $^{\mathrm{b}}$ \\ ${ }^{a}$ State-province Joint Engineering Laboratory of Spatial Information Technology for High-speed Railway Safety, Southwest \\ Jiaotong University, Chengdu, 611756 , P.R. China - lishen@ home.swjtu.edu.cn \\ ${ }^{\mathrm{b}}$ Faculty of Geosciences and Environmental Engineering, Southwest Jiaotong University, Chengdu, 611756, P.R. China - \\ lishen@home.swjtu.edu.cn, linmay23@yeah.net, zhipengliswjtu@ foxmail.com
}

Commission VII, WG VII/4

KEY WORDS: Topic modelling, Image classification, Object-based, Multiscale segmentation

\begin{abstract}
:
Multiscale segmentation is a key prerequisite step for object-based classification methods. However, it is often not possible to determine a sole optimal scale for the image to be classified because in many cases different geo-objects and even an identical geoobject may appear at different scales in one image. In this paper, an object-based classification method based on mutliscale segmentation results in the framework of topic modelling is proposed to classify VHR satellite images in an entirely unsupervised fashion. In the stage of topic modelling, grayscale histogram distributions for each geo-object class and each segment are learned in an unsupervised manner from multiscale segments. In the stage of classification, each segment is allocated a geo-object class label by the similarity comparison between the grayscale histogram distributions of each segment and each geo-object class. Experimental results show that the proposed method can perform better than the traditional methods based on topic modelling.
\end{abstract}

\section{INTRODUCTION}

Recent advances in remote sensor technology, particularly those relating to spatial resolution, are helping to make detailed observation of the earth's surface possible. However, the resulting huge amounts of very high resolution (VHR) satellite images pose a challenge for automatic classification, due to the large amount of information and within-class variance characteristics of this kind of images (Benediktsson et al, 2013).

It is widely acknowledged that compared to pixel-based methods, object-based classification methods are probably more appropriate for VHR satellite images (Blaschke, 2010). For object-based image analysis (OBIA), a key prerequisite step is to perform image segmentation, which aims to produce a set of non-overlapping segments. Multiscale segmentation is the most popular algorithm for this purpose. This kind of methods conduct a series of image segmentations at multiple scales in scale space and sometimes propagates from coarse to fine scales followed by selecting an optimal scale for classification task (Drăguţ et al, 2014). Although these methods have been widely used, there exist some problems in their practical applications: 1) the determination of an optimal scale is often difficult; 2) it is often not possible to solely determine one optimal scale for the image to be classified because in many cases different geoobjects and even an identical geo-object may appear at different scales in one image. These observations motivate us to develop a new OBIA approach based on multiscale segmentation results, in which multiple segmentations are jointly utilized by means of topic modeling.

In recent years, probabilistic topic models, e.g. probabilistic latent semantic analysis (pLSA) (Hofmann, 1999) and latent Dirichlet allocation (LDA) (Blei et al, 2003), have been successfully used in natural image analysis and satellite image understanding (Liénou et al, 2010 and Bratasanu et al, 2011). In the framework of these models, each document is viewed as a mixture of topics, and each topic has its corresponding specific distribution over words.

Yi proposed a novel semantic clustering algorithm for VHR satellite images using the pLSA model. The experiment results demonstrated the advantage of topic model based method (Yi et $a l$, 2011). However, a pre-processing step is required to partition a satellite image to image blocks and an accompanying post-processing step is required to combine all the allocated labels of a pixel into a unique one using certain rules. In order to overcome this shortcoming, Tang proposed an msLDA model, which builds an automatic framework that combines a latent Dirichlet allocation with a multiscale image representation of a panchromatic satellite image (Tang et al, 2013). The msLDA also archives an adaptive smoothing on clustering results. Nevertheless, its application usually introduces a computational bottleneck, due to the generation way of image documents. Shen proposed a semisupervsied latent Dirichlet allocation model by considering over-segments as documents (Shen et al, 2014). However, it also suffers from the same problem as the traditional object-based methods mentioned previously, i.e., it is modelled based on the segmentation map at one scale.

This paper presents a novel OBIA approach based on multiscale segmentation results using latent Dirichlet allocation model to classify VHR satellite images in an entirely unsupervised fashion. It holds the assumption that each segment in multiple segmentation layers should take one geo-object class, and each geo-object class should correspond to a specific distribution of visual words, i.e., the grayscale value of pixels. In the stage of

\footnotetext{
* Corresponding author
} 
topic modelling, grayscale histogram distributions for each geoobject class and each segment are learned in an unsupervised manner from multiscale segments. In the stage of classification, each segment is allocated a geo-object class label by the similarity comparison between the grayscale histogram distributions of each segment and each geo-object class (Russell et al, 2006).

The remainder of this paper is organized as follows. In Section 2, the proposed approach is presented in detail. Experimental results and some discussion are given in Section 3. Finally, the conclusion is drawn in Section 4.

\section{METHODOLOGY}

This section firstly defines correspondences of text-related terms in the image domain. Then the topic modelling utilizing a single segmentation map and multiple segmentation ones are presented, respectively.

\subsection{Build An Analogue of Text-related Terms in Image} Domain

To borrow techniques used in the text domain to satellite images, the most important issue that needs to be addressed is how to build an analogue of text terms in image domain. For the proposed method, we follow the definition in (Tang et al, 2013) and (Shen et al, 2014):

1) Word: the grayscale value of a pixel is defined as a word.

2) Vocabulary: the unique grayscale values of the satellite image are viewed as the vocabulary.

3) Document: an image segment is defined as a document.

4) Topic: a geo-object class is defined as a topic.

\subsection{Topic Modelling based on A Single Segmentation Map}

Figure 1 shows the flowchart of the proposed topic modelling algorithm for the satellite image classification task, as well as the traditional one (Yi et al, 2011).

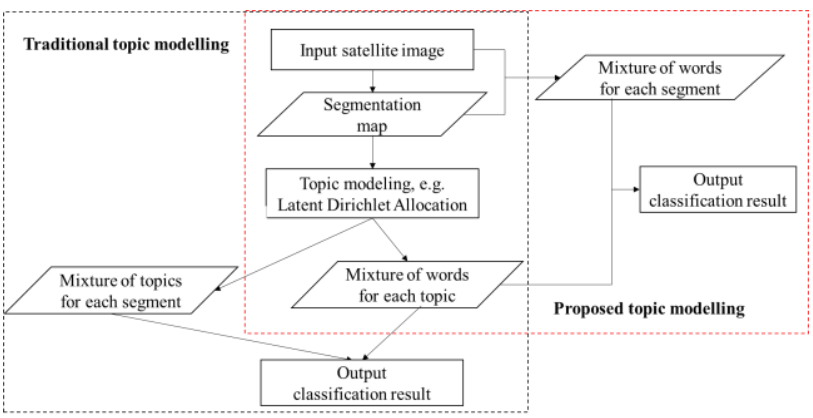

Figure 1. The flowchart of the traditional and proposed topic modelling algorithms for the satellite image classification

The processing procedure can be summarized in the following three steps.

1) Pre-processing: a segmentation map is firstly created by over-segmenting the original satellite image to constitute a corpus of documents. Then each segment is viewed as a bag of words (BOW).

2) Topic Modelling: the LDA model is utilized to conduct the topic discovery process. Figure 2 shows the graphic model of the LDA, in which $\alpha$ and $\beta$ are hyperparameters, $\theta$ follows a Dirichlet distribution of $\operatorname{Dirichlet}(\alpha)$ and $\phi$ follows $\operatorname{Dirichlet}(\beta)$.The goal of the topic modelling is to estimate the probability of picking the topic (geo-object class) $z_{k}$ in the document (segment) $d_{j}\left(\theta_{j}^{k}=p\left(z_{k} \mid d_{j}\right)\right)$ and the probability of generating the word $w_{i}$ (grayscale value) from the topic (geoobject class) $z_{k}\left(\phi_{k}^{i}=p\left(w_{i} \mid z_{k}\right)\right)$.

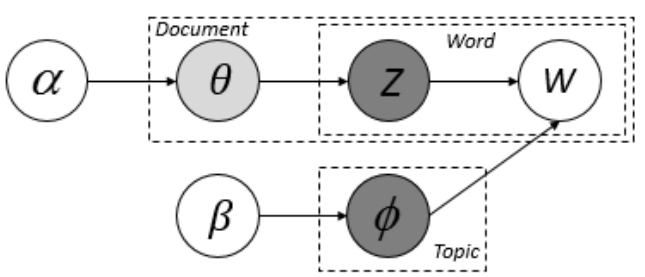

Figure 2. The graphic model of LDA

Gibbs sampling technique is often used to calculate the conditional probabilities $p\left(z_{k} \mid d_{j}\right)$ and $p\left(w_{i} \mid z_{k}\right)$.

$$
\begin{gathered}
\theta_{j}^{k}=\frac{N u m_{j}^{k}+\alpha_{k}}{\sum_{k^{\prime}=1}^{K} N u m_{j}^{k^{\prime}}+K \alpha_{k}}, \\
\phi_{k}^{i}=\frac{N u m_{k}^{i}+\beta_{i}}{\sum_{v^{\prime}=1}^{V} N u m_{k}^{v^{\prime}}+V \beta_{i}} .
\end{gathered}
$$

Here, $K$ is the number of topics (geo-object classes) and $V$ is the size of the vocabulary. $N u m_{j}^{k}$ denotes the number of words (grayscale value) corresponding to the topic (geo-object class) $k$ in the document (segment) $j$, and $N u m_{k}^{i}$ denotes the number of words (grayscale value) $i$ which topic (geo-object class) label is $k$.

3) Image Classification: for the traditional topic modelling algorithm, the geo-object class label of the pixel $i$ in the segment $j$ is determined by the posterior probability:

$$
\text { Topic }_{w_{i}}=\underset{1 \leq k \leq K}{\arg \max } p\left(z_{k} \mid d_{j}\right) * p\left(w_{i} \mid z_{k}\right) .
$$

As shown in Figure 1, the proposed method determines the geoobject class label of each segment by the similarity comparison between the grayscale histogram distributions of each segment and each geo-object class. Each segment is classified as a geoobject class label with the maximum similarity. KullbackLeibler (KL) divergence is effective to describe the similarity of two distributions (Russell et al, 2006). Therefore, in this paper, the KL divergence is used to estimate the similarity of the visual word (grayscale value) distribution between each segment and each geo-object class, i.e.,

$$
\text { Topic }_{\text {segment }_{m}}=\underset{1 \leq k \leq K}{\operatorname{argmin}} K L\left(p\left(w_{i} \mid \text { segment }_{m}\right), p\left(w_{i} \mid z_{k}\right)\right),
$$

where $p\left(w_{i} \mid\right.$ segment $\left._{m}\right)$ stands for the proportion of the words acquiring the grayscale value $w_{i}$ in the segment $m$, Topic segment $_{m}$ denotes the geo-class label of the segment $m$. The definition of $K L$ divergence is:

$$
K L\left(p\left(w_{i} \mid \operatorname{seg}\right), p\left(w_{i} \mid z_{k}\right)\right)=\sum_{i} p\left(w_{i} \mid \operatorname{seg}\right) * \frac{\log p\left(w_{i} \mid \operatorname{seg}\right)}{\log p\left(w_{i} \mid z_{k}\right)} .
$$




\subsection{Topic Modelling based on Multiscale Segmentation}

Due to the existence of multiple scale effect in satellite images, it is often not possible to solely determine one optimal scale for the image to be classified because in many cases different geoobjects and even an identical geo-object may appear at different scales in one image. Therefore, we integrate multiscale segmentation results into the framework of topic modelling.

As shown in Figure 3, the proposed method can be summarized in the following three steps.

1) First Part: producing multiple segmentation of the satellite image to construct a hierarchical corpus of documents.

2) Second Part: topic discovery step is conducted based on all layers of segments other than only one segmentation map.

3) Third Part: allocate geo-object class labels to the segments of the segmentation map at each scale. It should be noted that because the first part produces multiple segmentation maps, the final classification result will include a set of results at different scales.

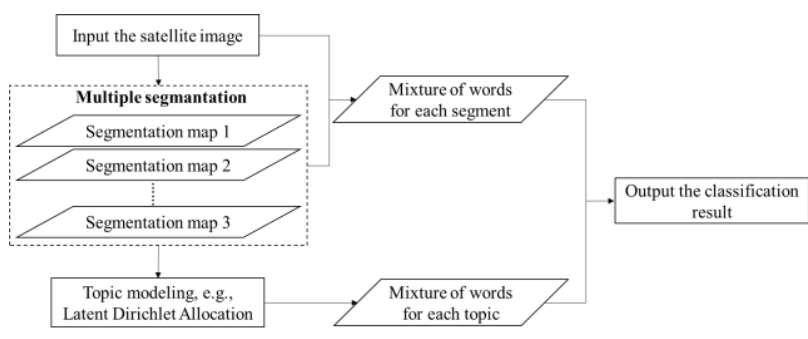

Figure 3 The proposed topic modelling algorithm based on multiscale segmentation

\section{EXPERIMENT RESULT}

\subsection{Experiment Data}

As shown in Figure 4(a), a panchromatic QuickBird image with $900 \times 900$ pixels and $0.6-\mathrm{m}$ spatial resolution is used, which is acquired on April 22, 2006 and located in in Tong Zhou district of Beijing, China. There are six main geo-object classes, including building, road, shadow, water, tree, and field. The corresponding ground truth image is shown in Figure 4(b).

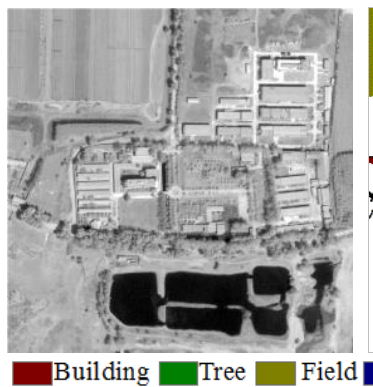

(a)

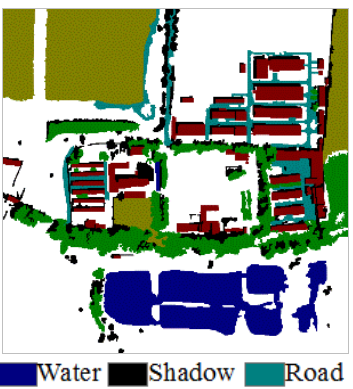

(b)
Figure 4 (a) The original panchromatic QuickBird image, (b) The ground truth map

\subsection{The Classification based on A Single Segmentation}

In order to evaluate the effectiveness of the proposed framework, we firstly compare the classification result of the proposed topic modelling based on a single segmentation with that of traditional one. The experiment uses the number of the segments to control the scale size for each segmentation map.
For this part, we set the number of the segments to 1500 (this number was experimentally derived to ensure the satellite image can be over-segmented) to over-segment the original image and Figure 5 shows the segmentation result about the original image.

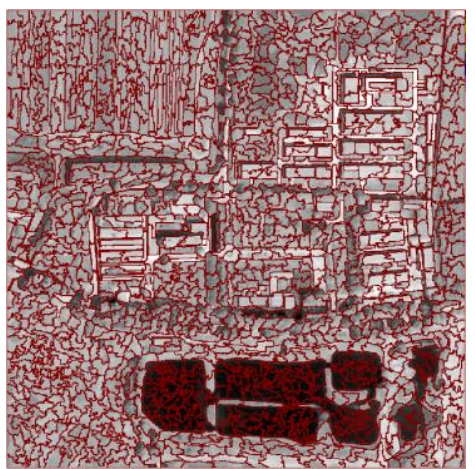

Figure 5 The segmentation result (the number of segments is set to 1500$)$

The hyperparameters $\alpha$ and $\beta$ of the topic modelling that we used in this paper are set to $15 / K$ and 0.5 , where $K=7$. Moreover, we run the Gibbs sampling during the topic modelling for 100 iterations. Figure 6 shows the conditional probability distribution $p\left(w_{i} \mid z_{k}\right)$ through topic modelling.

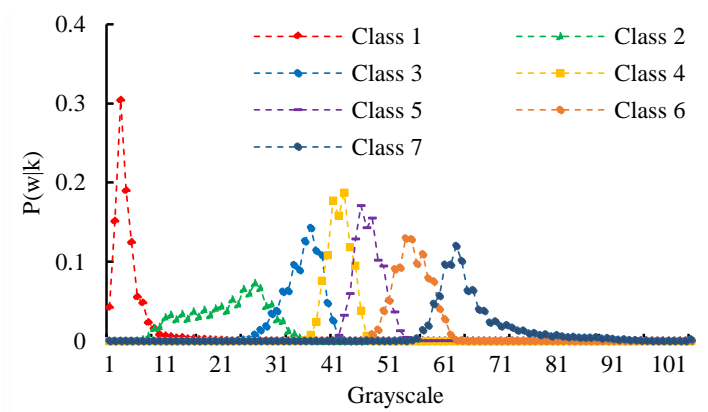

Figure 6 The probability distributions of the grayscale values given different geo-object classes

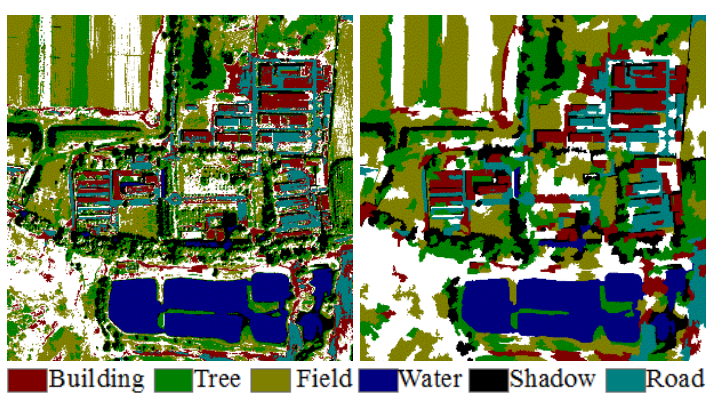

(a)

(b)

Figure 7 The classification results: (a) traditional topic modelling; (b) the proposed topic modelling

\begin{tabular}{|c|c|c|}
\hline & $\begin{array}{c}\text { Traditional topic } \\
\text { modelling }\end{array}$ & $\begin{array}{c}\text { The proposed } \\
\text { topic modelling }\end{array}$ \\
\hline Kappa coefficient & 0.6389 & 0.6542 \\
\hline Water & 0.9573 & 0.9689 \\
\hline Building & 0.5059 & 0.5675 \\
\hline Field & 0.6037 & 0.6340 \\
\hline
\end{tabular}




\begin{tabular}{|c|l|l|}
\hline Road & 0.5299 & 0.5682 \\
\hline Shadow & 0.7292 & 0.6027 \\
\hline Tree & 0.4097 & 0.4530 \\
\hline
\end{tabular}

Table 1. Classification accuracy for the two methods.

Figure 7 shows the different results of the two methods. From visual inspection, the proposed approach obtains a more compact and smoother classification result. Table 1 gives the classification accuracy. Both the global accuracy (Kappa coefficient) and the class-specific classification accuracy are presented. From the Table 1, we can draw that the classification accuracy of the proposed topic modelling algorithm is better that traditional one, though it achieved a less accuracy for shadow.

\subsection{The Influence of Segmentation Scale}

A key step for the proposed topic modelling is to conduct similarity comparison between the grayscale histogram distributions of each segment and each geo-object class. Therefore, we also need to take the influence of the scale effect into account.

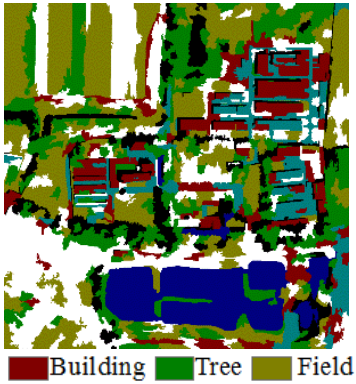

(a)

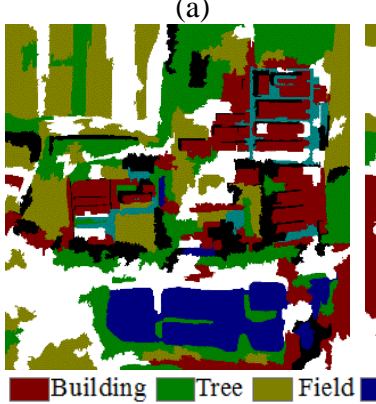

(c)

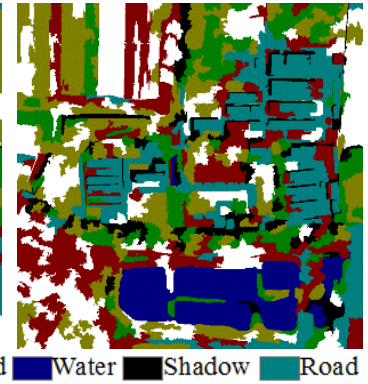

(b)

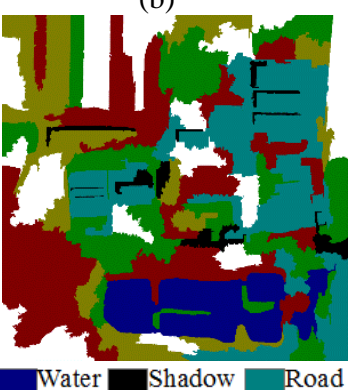

(d)
Figure 8 The different classification results from the segmentation map at different scales

A set of experiments with different image segmentation maps including different number of segments (i.e., 1500, 1000, 500 and 200), which can reflect the scale effect, is conducted. As shown in Figure 8, a larger number of segments results in a more heavily smoothed classification results. But it also filters the detailed information of certain geo-objects. Therefore, it is necessary to integrate multi-scale segmentation results into the learning of topic modelling.

\subsection{Classification Based on Multiple Segmentations}

Considering the different granularities of the geo-objects, e.g. the narrow roads may be suitable to be extracted at a fine scale, while the large-area field should be implemented classification at a coarse scale. Therefore, when processing the multiple segmentation of the original image, the scales chosen should meet the requirements of all granularities of the geo-objects. The simplest way is to conduct a series of image segmentations at multiple scales in scale space and sometimes propagates from coarse to fine scales. In this experiment, six scales, i.e., six numbers of segments $(1500,1000,500,200,100,50)$ is selected to segment the original satellite images. All layers of segments other than only one segmentation map are used to conduct topic modelling. Figure 9 shows the different classification results.

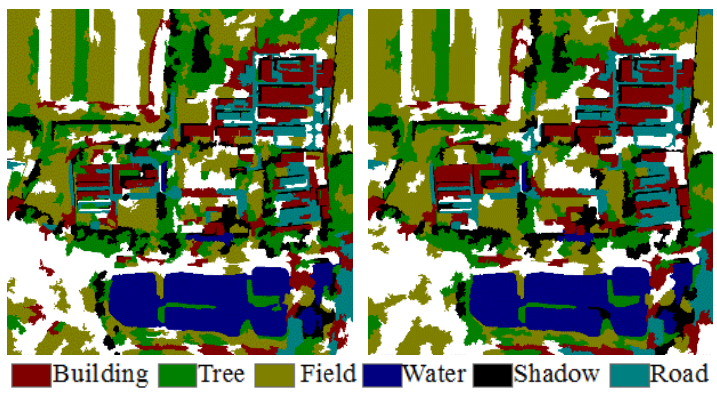

(a)
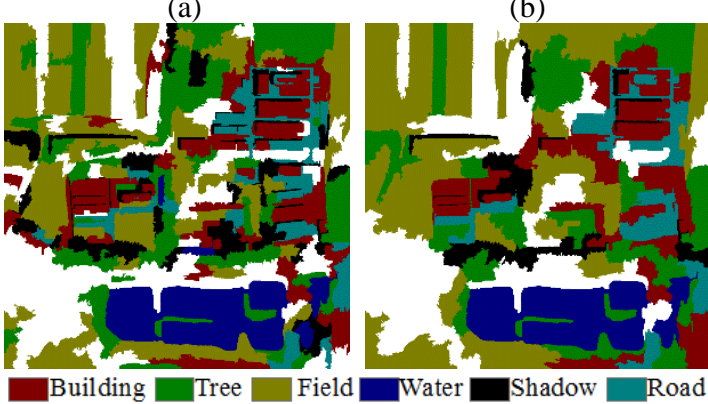

(c)

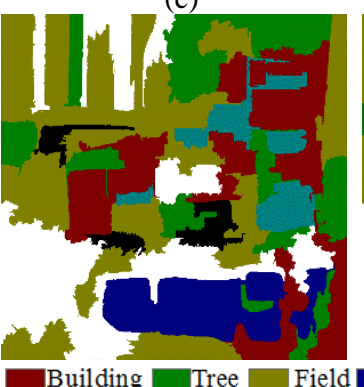

(e)

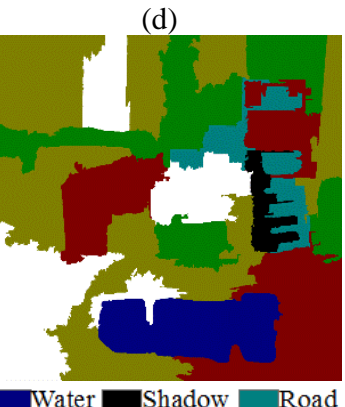

(f)
Figure 9. Classification results based on different segmentations (The number of segments for image segmentation is 1500,1000 , $500,200,100$, and 50, respectively)

Through comparing Figure 8 and Figure 9, e.g., Figure 8(b) and Figure 9(b), more smoothed classification results are achieved based on multiple segmentations in large-area field. However, in the current framework, the final classification result is still subject to the scale, e.g., the number of segments, which is used to finally determine the classification result. That is to say, although the topic modelling is learned based on multiple segmentation maps, the classification results are actually determined at different scales, respectively.

\section{CONCLUSION AND DISCUSSION}

In this paper, multiscale segmentation results are jointly used to conduct an object-based classification of VHR satellite images in the frame of topic modelling. Experiments prove the effectiveness of the proposed approach. 
Though a classification result with increased accuracy can be obtained in the current framework, an adaptive smoothing classification result cannot still be achieved. In order to solve this problem, we will further consider how to integrate the multiple classification results producing by learning multiscale segmentations to a unique one in the future.

\section{ACKNOWLEDGEMENTS}

This work was supported by the National Basic Research Program of China (No. 2012CB719901), the National Natural Science Foundation of China (No.41401374), and the Program for Changjiang Scholars and Innovative Research Team in University (No.IRT13092).

\section{REFERENCES}

Benediktsson, J.A., Chanussot, J., and Moon, W.M. Advances in very-high-resolution satellite. Proceedings of the IEEE, 2013, 101(3):566-569.

Blaschke, T. Object based image analysis for satellite. ISPRS Journal of Photogrammetry and Satellite, 2010, 65(1):2-16.

Blei, D. M., Ng, A. Y., and Jordan, M. I. Latent Dirichlet allocation. Journal of Machine Learning Research, 2003, 3:993-1022.

Bratasanu, D., Nedelcu, I., and Datcu, M.. Bridging the semantic gap for satellite image annotation and automatic mapping applications. IEEE Journal of Selected Topics in Applied Earth Observations and Remote Sensing, 2011, 4(1):193-204.

Drăguţ, L., Csillik, O., Eisank, C., et al. Automated parameterisation for multi-scale image segmentation on multiple layers. ISPRS Journal of Photogrammetry and Remote Sensing, 2014, 88:119-27.

Hofmann, T. Probabilistic latent semantic indexing. Proceedings of the 22nd Annual International ACM SIGIR Conference on Research and Development in Information Retrieval, 1999, 50-57.

Liénou, M., Maître, H., and Datcu, M. Semantic annotation of satellite images using latent Dirichlet allocation. IEEE Geoscience and Remote Sensing Letters, 2010, 7(1): 2832.

Russell, B. C., Freeman, W. T., Efros, A., et al. Using multiple segmentations to discover objects and their extent in image collections. Proceedings of 2006 IEEE Computer Society Conference on Computer Vision and Pattern Recognition, 2006, 1605-1614.

Shen, L., Tang, H., Chen, Y., et al. A semisupervised latent dirichlet allocation model for object-based classification of VHR panchromatic satellite images. IEEE Geoscience and Remote Sensing Letters, 2014,11(4):863-7.

Tang, H, Shen, L., Qi, Y., et al. A multiscale latent dirichlet allocation model for object-oriented clustering of VHR panchromatic satellite images. IEEE Transactions on Geoscience and Remote Sensing, 2013, 51(3):1680-92.

Yi, W., Tang, H., Chen, Y. An object-oriented semantic clustering algorithm for high-resolution satellite images using the aspect model. IEEE Geoscience and Remote Sensing Letters, 2011, 8(3):522-6. 